\title{
The Role of Neurous Autophagy in Pulmonary Encephalopathy and Signaling Pathways
}

J Han, F Zeng, S Cheng, Z Gong, Y Zhou

\begin{abstract}
Objective: To survey the effects of hypoxia on autophagy in the pulmonary encephalopathy.
\end{abstract}

Patients and Methods: A study on 50 patients of COPD complicated by pulmonaryencephalopathy (experimental group) and 50 patients of COPD without pulmonary encephalopathy (controls group). Cerebrospinal fluid of patients with lumbar puncture is acquired, $\beta$-glucocerebrosidase enzyme, ATG protein concentration were detected by ELISA and P62 protein concentration was detected by Western Blot.

Result: The results showed that the expression of the concentration of ATG7 protein and P62 protein and the $\beta$ - glucocerebrosidase enzyme in patients of COPD complicated by pulmonary encephalopathy was significantly higher than those of patients without pulmonary encephalopathy.

Conclusion: This study suggested that autophagy in the central neuronal system of patients with pulmonary encephalopathy enhanced. So we can observe neuron autophagy in the pulmonary encephalopathy induced by hypoxia, and accumulate information for the prevention and treatment of pulmonary encephalopathy.

Keywords: Autophagy, hypoxic, pulmonary encephalopathy, signal pathway

From: Department of Respiration Medicine, The First College of Clinical Medical Sciences, China Three Gorges University, Yichang 443000, Hubei Province, China.

E-mail address: 308605312@qq.com

Correspondence: Dr F Zeng, Department of Respiration Medicine, The First College of Clinical Medical Sciences, China Three Gorges University, Yichang 443000, Hubei Province, China

* Jiao Han and Fanjun Zeng contribute equally to this work. They are listed as co-first author 


\section{INTRODUCTION}

Chronic Obstructive Pulmonary Disease (COPD) is a major public health problem. It is the fourth leading cause of chronic morbidity and mortality in the United States and (1) is projected to rank fifth in 2020 as a worldwide burden of disease according to a study published by the World Bank/World Health Organization (2).

The tissue of brain of acute phase patients suffering from chronic pulmonary heart disease require a lot of oxygen, accounts for about 1/5 1/4 of oxygen consumption of the body. Cortical neurons is so sensitive to oxygen that depleted of oxygen for $4 \sim 5$ minutes can lead to irreversible nerve damage. The pathogenesis of pulmonary encephalopathy has not been fully elucidated, now think of oxygen and $\mathrm{CO} 2$ retention and acidosis are three basic elements of neuronal damage. During the development of pulmonary encephalopathy, there are several pathologies, such as autophagy apoptosis and necrosis. Apoptosis is the Representative of programmed cell death, called type I programmed cell death. Autophagy is a kind of programmed cell death that found after the apoptosis, called type II programmed cell death. So in addition to the necrosis and apoptosis, autophagy is classified as the third kind of forms of cell death.

Autophagy, also called type II programmed cell death, is a fundamental eukaryotic process with multiple cytoplasmic homeostatic roles. In animals and yeasts, autophagy is often divided into three main types: chaperone-mediated autophagy, microautophagy and macroautophagy. Macroautophagy is performed in the cytoplasm, whereby a portion of cytoplasm is sequestered inside a double-membrane-bound organelle (3). Macroautophagy the most common in autophagy, so, if no special instructions, all autophagy is refers to the 
macroautophagy (4).

Many studies about autophagy signaling pathways had been done, but the signaling pathways involved in the formation of autophagy is still quite complicated. Recently, there are mainly two pathways been surveyed: mTOR (mamma lian target of rapamycin) pathway and nonmTOR -dependent signaling pathway (5). Autophagy regulated mainly by the mTOR pathway in the case of ischemia and hypoxia.

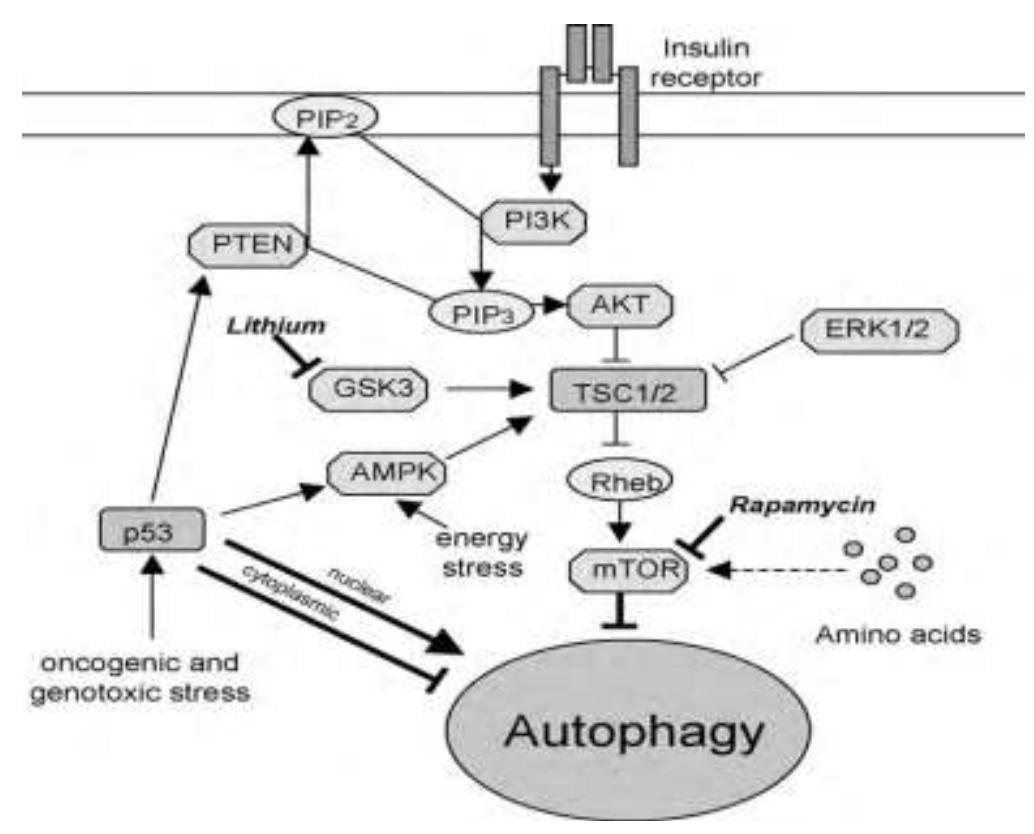

Fig 1: mTOR pathway

The formation of autophagy need the participation of ULK - 1 / Atg1 - Atg13 - FIP200 / Atg17 complex(6) (Fig. 1 ).When there is plenty of nutrition, TOR combined with ULK - 1 complex restrains autophagy. While when there is lack of nutrition, this complex dissociate and the ULK - 1 complex will promote the occurrence of autophagy. Thus it can be seen that the change of nutrition metabolism, growth factors and energy situation can inhibit or activate 
mTOR and determine whether autophagy occurs or not.

Raptor main recognises molecule TOR (TOR signaling, TOS) motif on the substrate. Rapamycin induce autophagy by interfering with raptor's recognizing S6K1 and inhibiting the phosphorylation of S6K1.But mTORC2 is not sensitive to rapamycin. Class I PI3K/Akt signaling pathway, MAPK/Erk1/2 signaling pathways and AMPK - mTOR pathway can activate the mTOR finally (7) (Fig. 1 ).

In the condition of ischemia and hypoxia, such as hunger, the occurrence of autophagy is mainly composed of mTOR pathway. The signal of hypoxia makes mTOR signaling pathways inactivation, and activate autophagy signaling pathways. Short-term ischemia hypoxia can strengthen the autophagy and apoptosis in nerve cell of the newly born mouse. But if autophagy degrade intracellular proteins and organelles exceedingly, the ATP in cells will be excessive consumed, and then the cells will death in the end.

\section{PATIENTS AND METHODS}

Selected 50 patients who are diagnosed as COPD and other 50 patients who are diagnosed as COPD complicated by pulmonary encephalopathy in the central hospital of Yichang City, Hubei Province, China, from January 2012 to January 2013. Then attribute them to COPD + pulmonary encephalopathy group (study group) and COPD group (control group) .The patients must exclude other diseases, such as tuberculous pleurisy, viral or parasitic infection, cancer and so on. Collect cerebrospinal fluid of the two groups by lumbar puncture. After centrifugal separation, the $\beta$-glucocerebrosidase enzyme, ATG protein and P62 protein can be 
gathered. Then detect $\beta$-glucocerebrosidase enzyme and ATG protein by ELISA and detect P62 protein by Western Blot. In the end, establish a standard curve using BCA protein quantification method.

\section{RESULTS}

\section{Expression levels of $\mathbf{P 6 2}$ in the two groups}

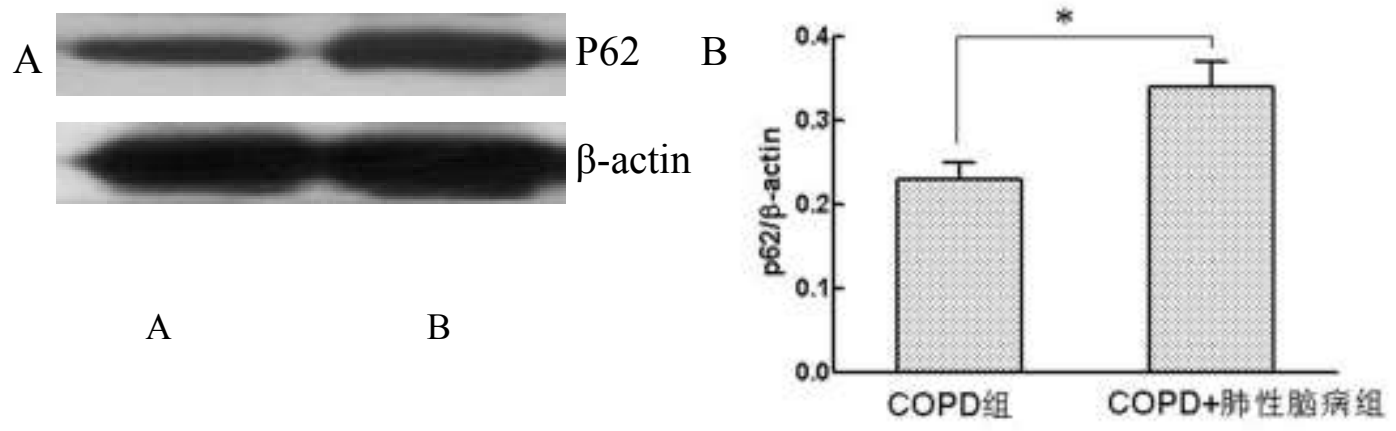

Fig 2: Expression levels of P62 in the CSF of the two groups is detected by Western blot. A: control group, B: study group.

P62 is closely related to autophagy. P62 is an LC3 interaction partner in vivo and is constantly degraded by autophagy, establishing p62 as a useful marker for autophagic vesicle turnover. It degrade in the mid or late autophagic (8). In general, there is a negative correlation between intracellular P62 expression and the activity of autophagic. Mizushima has observed that P62 accumulate in the condition of deficient in autophagy induced by 
hungry(9).A study report that the expression level of P62 has a close relationship with many autophagy-related factors(10), but autophagy is not the only degradation pathway for P62(11).

To characterize the expression levels of P62 of the two groups we performed a series Western blot. It showed that the expression of the concentration of P62 protein in the CSF of COPD complicated by pulmonary encephalopathy patients is much higher than that of COPD patients. (Fig. 2 A) The gray vanue of the two groups show that there is a significant difference to the two groups. $(\mathrm{P}<0.05)$ (Fig. 2 B).From this study we can conclude that autophagy in the cortical neurons of COPD complicated by pulmonary encephalopathy patients has enhanced. This can improve the tolerance of neurons in the condition of hypoxia and $\mathrm{CO} 2$ retention. Thus autophagy can be a protector for neurons.

Table: Expression levels of $\beta$-glucocerebrosidase enzyme in the two group

\begin{tabular}{llll}
\hline Group & $\mathrm{n}$ & $\begin{array}{l}\beta-\text { glucocerebrosidase } \\
\text { enzyme }\end{array}$ & ATG7 \\
\hline Control group & 50 & $15.5 \pm 3.6$ & $2.8 \pm 0.6$ \\
experimental group & 50 & $34.6 \pm 6.8^{*}$ & $12.4 \pm 3.2^{\triangle}$ \\
\hline
\end{tabular}

Expression levels of $\beta$ - glucocerebrosidase enzyme and ATG7 in the CSF of the two groups is detected by ELISA. After the test, $* \mathrm{P}<0.01$ vs Control group, $\Delta \mathrm{P}<0.05$ vs experimental group

Glucocerebrosidase (GBA) is a kind of hydrolase, it present in lysosomes and its molecular weight of 59700 Daltons. At first, it was found to be connected with Gaucher disease. Gene mutation lead to the decrease and lack of GBA, thus the glucocerebroside in macrophage can not be hydrolyzed and cumulate in the lysosomes. As a consequence, the function of the cells are disturbed. Later, another survey report it has relationship with Parkinson's disease (12).In this study we can see that the expression levels of $\beta$ glucocerebrosidase enzyme in the CSF of experimental group is much higher than that of control group. $(\mathrm{P}<0.05)$ [Table]. We can speculated that GBA associated with pulmonary encephalopathy. Although autophagy has relationship with lysosomes and pulmonary encephalopathy, whether it has a direct or indirect relationship with GBA is still uncertain 


\section{Expression levels of ATG7 enzyme in the two group}

Recent study showed that at least sixteen kind of Atg genes participate in the formation of autophagy. And Atg1, which was the first autophagy gene cloned successfully (13), is a mesentery gene in the formation of autophagy. Like ULK1, one of the homologous proteins in mammals, Atg1 encodes a kinase called Serine/threonine protein kinase. When there is lack of ULK1 kinase, which can regulate and control the formation of LC3-II, the formation of autophagy will be restrained (14).Being regulated by regulatory factor mTOR of the center of the autophagy ,ULK1 exists in the form of compound.

Of the many ATG genes, seven uniquely compose two ubiquitin-like conjugation systems: ATG12 and ATG8 conjugation systems. The ubiquitin-like protein Atg12p covalently attaches to Atg5p in a reaction similar to ubiquitination. In this process, Atg12p is activated by an E1-like enzyme, Atg7p, and transferred to an E2-like enzyme, Atg10p, and then finally conjugates to Atg5p. Atg8p, another ubiquitin-like protein, is unique among other ubiquitin-like molecules, as it conjugates to phosphatidyl-ethanolamine. Atg8p is activated by Atg7p, which is common to the Atg12 conjugation system, and is transferred to Atg3p, an E2-like enzyme. In mammals, there exist at least three Atg8 homologues that can all be activated by Atg7, GATE-16,GABARAP, and LC3, and they localize to the autophagosome(15).A report observed that when blocking Atg7p expression inside the L929 cell of mice, the incidence rate of autophagic cell death reduced sharply(16).

By comparing the expression levels of ATG7 in the CSF of the two groups, we observed that the expression of the concentration of ATG7 protein in patients of COPD 
complicated by pulmonary encephalopathy was much higher than those of patients without pulmonary encephalopathy $(\mathrm{P}<0.05)$ (Fig. 3). The result might be because a part of neurons cracked after the neurons been damaged, and a lot of Atg7p was released into the arachnoid membrane.

\section{DISCUSSION}

Pulmonary encephalopathy is one of the disorders of the central nervous system caused by respiratory failure, it depends mainly on the level of $\mathrm{PaCO} 2$ and $\mathrm{PH}$ value. Hypoxia is the core factor for pulmonary encephalopathy. Neuronal hypoxia can cause autophagic death or apoptosis. Autophagy has long been considered to be protected to neuronal. In the case of ischemia and hypoxia and other stress, autophagy plays a significant role in promoting cell survival, removing the aging organelles of cells and it is also important for the misfolded proteins. But other surveys show that autophagy lead to cells death under certain conditions. Autophagy is being tightly regulated and controlled as one of the important ways of metabolism.

In this study, because of the ethical issues, sample into the non-blank control group. Although because of ethics, the design of the study that the experiment group is less affected the results in a certain extent, the results still have a certain credibility on evidence-based medicine. 


\section{CONCLUSION}

To sum up, the study confirmed that the concentration of the ATG7 protein and P62 protein and the $\beta$ - glucocerebrosidase enzyme in the CSF of the patients of pulmonary encephalopathy increase. Atg7 and the ubiquitin-like conjugation it participate in may be an important channel of the formation of autophagy in neurons of patients with pulmonary encephalopathy. Through this research, we can understand the molecular mechanisms of the formation of autophagy in neurons of patients with pulmonary encephalopathy, which may be new targets for diagnosis and treatment of pulmonary encephalopathy.

The authors have no financial relationships to disclose and no conflicts of interest to report.

Abbreviations

COPD — chronic obstructive pulmonary diseases;

ATG — autophagy-related gene;

CSF — cerebral spinal fluid. 


\section{REFERENCES}

1. Vlahos R, Bozinovski S (2014). Recent advances in pre-clinical mouse models of COPD. Clin Sci (Lond) 126: 253-265.

2. Ghasemi H, Owlia P, Jalali-Nadoushan MR, Pourfarzam S, Azimi G, et al. (2013). A clinicopathological approach to sulfur mustard-induced organ complications: a major review. Cutan Ocul Toxicol 32: 304-324.

3. van Doorn WG, Papini A (2013) Ultrastructure of autophagy in plant cells: A review. Autophagy 9.

4. Deretic V (2012) Autophagy: an emerging immunological paradigm. J Immunol 189: 15-20.

5. Tiwari RV, Parajuli P, Sylvester PW (2013) gamma-Tocotrienol-induced autophagy in malignant mammary cancer cells. Exp Biol Med (Maywood).

6. Crotzer VL, Blum JS (2005) Autophagy and intracellular surveillance: Modulating MHC class II antigen presentation with stress. Proc Natl Acad Sci USA 102: 7779-7780.

7. Alers S, Loffler AS, Wesselborg S, Stork B (2012) Role of AMPK-mTOR-Ulk1/2 in the regulation of autophagy: cross talk, shortcuts, and feedbacks. Mol Cell Biol 32: $2-11$.

8. Kirkin V, McEwan DG, Novak I, Dikic I (2009) A role for ubiquitin in selective autophagy. Mol Cell 34: 259-269.

9. Mizushima N, Yoshimori T (2007) How to interpret LC3 immunoblotting. Autophagy 3: 542-545. 
10. Bjorkoy G, Lamark T, Brech A, Outzen H, Perander M, et al. (2005) p62/SQSTM1 forms protein aggregates degraded by autophagy and has a protective effect on huntingtin-induced cell death. J Cell Biol 171: 603-614.

11. Moskaluk C, Bastia D (1988) DNA bending is induced in an enhancer by the DNA-binding domain of the bovine papillomavirus E2 protein. Proc Natl Acad Sci USA 85: 1826-1830.

12. Lwin A, Orvisky E, Goker-Alpan O, LaMarca ME, Sidransky E (2004) Glucocerebrosidase mutations in subjects with parkinsonism. Molecular Genetics and Metabolism 81: 70-73.

13. Matsuura A, Tsukada M, Wada Y, Ohsumi Y (1997) Apg1p, a novel protein kinase required for the autophagic process in Saccharomyces cerevisiae. Gene 192: 245-250.

14. Jung CH, Jun CB, Ro SH, Kim YM, Otto NM, et al. (2009) ULK-Atg13-FIP200 complexes mediate mTOR signaling to the autophagy machinery. Mol Biol Cell 20: 1992-2003.

15. Komatsu M, Waguri S, Ueno T, Iwata J, Murata S, et al. (2005) Impairment of starvation-induced and constitutive autophagy in Atg7-deficient mice. J Cell Biol 169: 425-434.

16. Yu L, Alva A, Su H, Dutt P, Freundt E, et al. (2004) Regulation of an ATG7-beclin 1 program of autophagic cell death by caspase-8. Science 304: 1500-1502. 